\title{
Towards modelling the human nervous system in a dish: recent progress and outstanding challenges
}

\author{
Barbora Vagaska and Patrizia Ferretti ${ }^{\star}$ \\ Stem Cell and Regenerative Medicine Section, UCL Great Ormond Street Institute of Child Health, \\ University College London, 30 Guilford Street, London WC1N 1EH, UK
}

Corresponding author:

Prof. Patrizia Ferretti

Stem Cell and Regenerative Medicine Section

UCL Great Ormond Street Institute of Child Health, University College London

30 Guilford Street

London WC1N 1EH

UK

Email: p.ferretti@ucl.ac.uk 


\section{Executive Summary}

- Investigation of mechanisms governing development and normal and abnormal function of the human central nervous system (CNS) is hampered by its complexity and the very limited possibility of experimentally manipulating it in-vivo.

- Development of 3-dimensional (3D), tissue-like culture systems offers much promise for boosting our understanding of human neural development, birth defects, neurodegenerative diseases and neural injury

- 3D models of the human CNS can provide platforms that will allow us to more accurately predict efficacy of putative therapeutic compounds and assess responses to potentially neurotoxic agents.

- Novel technological developments and a more interdisciplinary approach to modelling the human CNS underpin development of these model

- As we increase the complexity of in-vitro systems to make them more tissue-like, the challenge of establishing highly reproducible models amenable to quantitative and high throughput analysis also increases. 


\begin{abstract}
Studying the cellular and molecular basis governing development and normal and abnormal function of the human central nervous system (hCNS) is hampered by its complexity and the very limited possibility of experimentally manipulating it in-vivo. Development of 3dimensional, tissue-like culture systems offers much promise for boosting our understanding of human neural development, birth defects, neurodegenerative diseases and neural injury, and for providing platforms that will more accurately predict efficacy of putative therapeutic compounds and assess responses to potentially neurotoxic agents. While novel technological developments and a more interdisciplinary approach to modelling the hCNS are accelerating the pace of discovery, increasing the complexity of in-vitro systems increases the ordeals to be overcome to establish highly reproducible models amenable to quantitative analysis.
\end{abstract}

Keywords: central nervous system, development, disease, human, in vitro modelling, stem cells 


\section{Article}

The central nervous system (CNS), including brain and spinal cord, is the most complex tissue in the body, both structurally and functionally. The main CNS components are the neurons and their extensions, glial cells (oligodendrocytes and astrocytes), vascular cells, immune cells and other support cells. The human brain contains approximately 100 billion neurons and a typical neuron has about 5000-200,000 synapses [1]. Finely tuned interactions between all different cell types through both physical and biochemical mechanisms lead to a highly sensitive and well-regulated environment [2]. The complexity of the human brain together with the limited possibility of experimentally manipulating it in vivo, however, poses major challenges to study mechanisms governing human neural development, neurodegenerative diseases and responses to injury at the cellular and molecular level. The results of animal studies, though of crucial importance to provide insights in some of these mechanisms, cannot be simply extrapolated to humans. Rodents have reduced cognition, less complex brain structure and transgenic animal models of neural diseases often lack features of the pathological phenotype observed in humans; examples of human neurological diseases that are challenging to model in animals include amyotrophic lateral sclerosis (ALS), Huntington disease and Parkinson disease and autism [3-8]. Furthermore, some of the species that might better recapitulate certain human neurological diseases are not traditional laboratory animals; hence, in addition to ethical issues and the high cost involved working with these models, there is a lack of suitable reagents available for their effective in depth study $[4,5,9]$. There are some significant differences between humans and rodents also at the cellular and molecular level; for example, rodent cells have longer telomeres that can account for differences in the severity of disease phenotype as compared to humans, and can respond differently to growth factors, cytokines and several toxic agents [10-15].

Hence it is not all that surprising that many CNS-targeted therapeutics that offer much promise in animal models of neural diseases fail in clinical trials. A key challenge is to develop tools to bridge this gap in order to improve our understanding of healthy and diseased human CNS at the cellular and molecular levels, and better predict efficacy of novel therapeutic strategies in humans. [16]. Because of the obvious ethical constraints, this has to be achieved by modelling the human CNS in vitro. 


\section{“Old" and" new” cell sources for human neural tissue modelling}

In vitro models, that have largely been 2-dimensional (2D, monolayers), significantly reduce the complexity of the whole organism into a more defined and controlled system, allow easy manipulation of both cells and their environment and thus allow us to study phenomena that cannot be observed or measured in vivo.

Because of the limited availability of human tissue and the need for large quantities of material, especially for biochemical and pharmacological studies, human neural cell lines have been much used in neuroscience. Many commonly used human cell lines were derived from naturally occurring tumours such as astrocytomas (e.g.U-87, U-251), oligodendrogliomas (e.g. HOG), ependymomas, glioblastomas, and primitive neuroectodermal tumors (e.g. TERA-2 and its subclone, the NT-2 line) [17]. Neuroblastomas, child solid tumours composed of neural crest derived precursors of the sympathetic nervous system, have been also extensively used as an easily available source of neuronal-like cells (e.g. SH-SY5Y) as following differentiation they display many feature of dopaminergic neurons [18]. In general, transformed human neural lines maintain some of the properties of native cells; therefore, as long as these models' limitations are taken into account, they can provide useful tools particularly for cellular and molecular studies (e.g. investigation of signalling pathways).

The use of non-tumor-derived human neural cells has significantly gained momentum over the last couple of decades following the discovery of neural stem cell presence not only in the developing CNS but also postnatally, and the development of strategies for their isolation, long-term culture and differentiation into neurones and glia (astrocytes and oligodendrocytes). This has gone hand in hand with developing protocols to generate different neural cell types from pluripotent human embryonic stem cells (ESCs). ESCs have self-renewal ability and readily proliferate, thus allowing generation of neuronal and glial cells in relatively large numbers [16, 19]. However, apart from the ethical issues concerning the use of ESC, these cells are usually derived from supposedly normal embryos. While they can be very valuable for assessing toxicity to different neural and glial populations [20], they are not directly suitable for disease modelling unless modified to carry mutations of interest when the disease cause is known. In the last few years, modelling of human neural diseases has been galvanized by the development of reprogramming techniques that allow generation of induced pluripotent stem cells (iPSCs) from patients' somatic cells (e.g. skin fibroblasts, blood cells) which can be also valuable for autologous stem cell therapy [21, 22]. iPSCs have ESC-like properties and their use circumvents ethical issues. Their downside is that reprogramming is a lengthy and costly 
process and that cell from patients are not always readily available to researchers. Several iPSC lines from patients with a number of neural diseases (e.g. spinal muscular atrophy, amyotrophic lateral sclerosis, Alzheimer's disease, ataxia telangiectasia, Down syndrome, familial dysautonomia, fragile X syndrome, Friedrich ataxia, Huntington's disease, Niemann-Pick disease, Parkinson's disease, schizophrenia) have already been generated and their number is continuously increasing [23]. Significantly, the number of open-access repositories of cells from patients carrying a variety of diseases is also rapidly increasing, with some focusing on neural diseases, but more are needed [24, 25]. It will be crucial to ensure long-term funding for maintenance and expansion of these important resources and guarantee their availability to the wider scientific community.

An example of how an iPSC-based model can be relevant to studying a disease and for therapeutic development is that of amyotrophic lateral sclerosis (ALS). This fatal disease is caused by mutations in superoxide dismutase 1 (SOD1) that lead to spinal neurone loss with consequent muscle wasting and paralysis. Currently, the only drug available to ALS patients merely delays the onset of some disease traits. The discovery of new drugs for ALS has been hampered by the lack of suitable models for preclinical testing as rodent models do not entirely mimic the human disease phenotype $[3,5]$. Neurones from ALS patient-derived iPSCs were found to display disease characteristics and have provided valuable insights into the activity of different drugs [25].

\section{Moving towards neural tissue-like structures}

Together, iPSCs provide a unique tool for studying human neural diseases for which there are no transgenic animal models fully reproducing the disease phenotype [16]. However, they have so far been mainly used in 2D cultures. While such cultures allow easy expansion, monitoring, and manipulation of cells, they also impose highly unnatural constraints on the cells. Cell behaviour, morphology and gene expression under these conditions can differ significantly from those observed in vivo. In addition, cells grown as monolayers often appear to be more susceptible to drugs and therapeutic agents ([26-28] and our paper in preparation). With increasing awareness of the importance of the environmental context for cell physiology, interest is growing in developing more complex cell culture systems that better reflect the situation in vivo and thus allow the cells to behave in a physiologically more relevant manner. To achieve this goal key properties of tissues need to be reproduced as closely as possible in the in vitro models (Figure 1). 
Development of three-dimensional (3D) human culture models can bridge the gap between native tissue and monolayer cell culture systems and increase the relevance of in vitro studies. A 3D architecture reduces the strain and artificial responses cells undergo to adapt to a flat surface as it allows them to spread and interact with adjacent cells in all dimensions. This results in different modulation of microenvironmental cues such as cell-cell and cell-matrix interactions, mechanical forces and diffusible factors providing a more natural environment that supports formation of a tissue-like architecture and can allow tackling questions that cannot be addressed in 2D systems [29, 30]. For example, modelling human CNS traumatic injuries using compressive impacts could not be achieved in 2D cultures [31].

The idea that $3 \mathrm{D}$ is better than $2 \mathrm{D}$ is not new. The oldest complex $3 \mathrm{D}$ systems used in neuroscience are organotypic cultures (derived from small explants or slices) where the cytoarchitecture of the tissue is largely maintained. Thin slices of brain or spinal cord can be kept alive for extended periods of time allowing cell labelling, manipulations, and live cell monitoring, including electrophysiological measurements. However, they are technically demanding, can be very variable and, in the case of human neural tissue, there is obviously limited tissue availability. Nonetheless, in a few studies, neural tissue from human foetal brain or spinal cord culture was used to study human CNS development [17, 32, 33] and more recently post-mortem human brain slices were shown to be suitable for optogenetic studies [34].

Nevertheless, as discussed above, if we are to model neural diseases, either patient-derived iPSCs, or ESCs or neural stem cells modified to carry the defective disease causing gene need to be used, and conditions that will give rise to a neural-like tissue in culture established.

The 3D model of choice, the complexity one aims to achieve and the strategy to be used to build it will depend on the specific questions to be addressed using that model. For example, when models are needed for studying mechanisms of human CNS development and disease, it can be acceptable to use systems that will slowly become organised into neural-like tissues and use quite complex and relatively time-consuming analytical approaches. In contrast, if a system is to be used for initial drug screening, it needs to be relatively easy to handle, inexpensive, highly reproducible, and able to provide clear readouts with medium to high throughput. 
Although the notion of bottom-up and top-down approaches is more commonly used in the context of tissue engineering, these approaches, either alone or in combination, can also be broadly applied to the construction of neural models [16].

In the bottom-up approach a model is put together from individual components to give rise to a more complex system. With this approach, cellular (e.g., phenotypes, ratios, densities) scaffold parameters (controlling mechanical properties and bioactive motifs) and construction approach (e.g. bioprinting) are defined to design and build the model in as controlled fashion as possible. They usually involve the use of a scaffold, and the type of scaffold selected depends on the desired characteristics and functionality of the 3D culture and on the method chosen to "build the tissue". For example, a relatively simple 3D model involves the use of rigid polystyrene scaffolds where human NSCs can be grown and induced to differentiate into mature neurons that form active and functional neuronal networks [35]. Hydrogels rich in brain extracellular matrix proteins, such as Matrigel, are extensively used to "glue" cells together in a 3D environment; as these scaffolds are not rigid, they allow for remodelling and are used also for top-down modelling. To give an example, Matrigel-embedded human NSCs overexpressing mutated human $\beta$-amyloid precursor protein and presenilin 1 have been used to establish a model of Alzheimer's disease. Upon induction of neuronal differentiation, these cells successfully recapitulated amyloid- $\beta$ and tau pathology with robust extracellular deposition of amyloid- $\beta$ plaques [36]. This model of Alzheimer's disease, as well models of other human neurodegenerative disorders developed using a similar strategy, will be valuable both for better understanding disease mechanisms and for therapeutics development.

The bottom-up approach could also allow generation of neural tissue of greater complexity by modular and patterned inclusion in the selected scaffold of different cell types (e.g. neurones, astrocytes, oligodendrocytes, microglia and endothelial cells) to interrogate their roles and interactions in biological processes. For example, it is difficult to study myelination and interactions between oligodendrocytes, the myelin-forming cells in the CNS, and neurons in the human developing brain, as it is currently hard to achieve efficient differentiation of hNSCs into oligodendrocytes and significant myelination in vitro. This is a bit of a vicious circle and highlights the inadequacy of the models used, as much information on the mechanisms regulating this process in the human brain is still missing, and myelination is a very "spatial" process that requires a 3D system. It is becoming apparent from animal studies that the developmental age of the oligodendrocytes and the presence of astrocytes may be key to efficient myelination [37]. This could be tested by systematically building human 3D models 
of different cellular composition. Availability of robust 3D human myelination models is crucial if we are to better understand its development, homeostasis and the process of demyelination-remyelination in neurodegenerative diseases such as multiple sclerosis (MS), as well as in response to injury.

One of the newest technologies applied in the bottom-up approach is 3D bioprinting, where complex 3D structures are fabricated through layer-by-layer precise positioning of biological materials, biochemicals and living cells, with very accurate spatial control of the placement of functional components. Bioprinting also allows high-throughput generation of replicas of spatially and temporally well-controlled complex constructs [38, 39]. The first example of fabricating human neural tissue by $3 \mathrm{D}$ bioprinting neural stem cells has been recently reported [40]; this opens new avenues to the development of more finely controlled and reproducible neural in vitro models.

The top-down approach to 3D modelling largely relies on the intrinsic ability of the cells to form complex structures, called organoids, usually within soft matrices [41, 42]. Cortex-like structures have been generated by allowing either a mixture of cells derived from human fetal brain or ESCs to self-organize in 3D usually within a hydrogel [42-44]. Well-controlled 3D aggregates of human ESCs spontaneously differentiated into cortical progenitors and functional neurons forming concentric patterned structures that mimicked early stages of corticogenesis and expressed markers of different cortical layers [42, 45-48] and references within). The self organising ability of human ESCs has indeed been demonstrated in several subsequent studies showing formation of cortex-like neuroepithelial cell layers, entire optic cups (primitive eye structures) [49], and stratified retinas [42, 46-48, 50]. A limiting factor for successful long-term maintenance and maturation of organoids is nutrient availability. By using a spinning bioreactor to enhance nutrient absorption, ESCs in Matrigel droplets can be maintained in culture for months (up to 10 months), allowing formation of brain organoids containing discrete regions that resemble cerebral cortex, ventral telencephalon, choroid plexus, retina and meninges [45]. The same approach was used to generate brain organoids from microcephaly patient-specific iPSCs to model this neurodevelopmental disorder in vitro [45]. The use of iPSC-derived neural organoids is rapidly expanding, and they have been recently used to study autism spectrum disorder, to model the effect of Zika virus infection, and to investigate evolutionary mechanisms underlying expansion of the human cortex, just to give some examples [46-48]. 


\section{Challenges ahead}

Notwithstanding all these exciting developments, establishment of highly reproducible 3D neural models is still in its infancy and presents several challenges (Figure 2). First of all the brain tissue has very high cellularity with little ECM. The average cell density in the brain is thought to exceed 100,000 cells/ $\mu \mathrm{L}$. Cell density has been shown to influence cell behaviour, including survival and differentiation [2]. Most culture systems cannot accurately reproduce this high cell density that is usually an order of magnitude lower than that found in vivo [51]. As already mentioned, high cellular densities raise the issue of adequate nutrient supply. Passive diffusion of nutrients and gasses is not sufficient to meet the metabolic demands of these dense cultures and further development of bioreactors providing continuous medium perfusion will be necessary [52]. Another approach to address these difficulties is miniaturization of the whole system using microfluidic devices [46]. Miniaturized culture chambers can provide high-resolution spatiotemporal control of the extracellular environment and reduce the number of cells and expensive reagents required. A number of models mimicking hypoxic neural stem niche, neuron-glia interactions, neurovascular unit and blood brain barrier, and culture of cells under dynamic interstitial fluid flow conditions have already been developed [20, 53-57]. In combination with 3D cultures "brain on chip" platforms offer a great system for studying physiology, injury or development at a single cell resolution as well as cytotoxicity or drug screenings.

Another big challenge in working with 3D culture models, that currently hinders their ease of use, is the lack of simple and reliable assays for detailed analysis and interpretation of cellular responses. Evaluation of basic parameters such as cell number, viability, morphology and functional testing is much more complicated in a 3D cell culture setting, particularly as its size increases, as compared to a 2D system. Development of robust, yet reasonably simple, methods for live cell imaging and functional evaluation of 3D in vitro models, including analysis of metabolic activities and electrophysiological responses, will be crucial. Without a thorough assessment of their properties, 3D models cannot be validated as true in vitro "neural tissue surrogates". Further development of micro-electrode array (MEA) technology could offer a solution to this problem and underpin development of the next-generation of 3D neural culture models. $[16,35,58]$.

Together, future development of reproducible and cost-effective human 3D models suitable for assessing mechanisms of neural development and disease, efficacy of therapeutic agents, or toxic responses, will require an extensive and well-integrated multidisciplinary effort [59, 60]. 
Although further refinement of 3D neural culture systems is much needed, it is already apparent that this in vitro approach can provide more tissue-like and physiologically relevant models for the study of the healthy and diseased human CNS, and help to increase accuracy in the evaluation of potential therapeutics, while reducing the need for animal studies.

Finally, the availability of physiologically relevant 3D neural culture models, together with a better understanding of neural disease mechanisms and identification of novel neural tissuecompatible scaffolds, might aid development of a diverse range of therapeutic approaches. For example, it could provide valuable references for the development and testing of novel matrices that can fill brain cavities following traumatic injury or stroke and be cellularised by endogenous cells [61-64], or aid devising cell-based systems for the delivery of neuroprotective factors in diseased brains [65].

\section{Future Perspective}

Development of human neural disease 3D models is taking momentum. It is anticipated that over the next few years interdisciplinary effort between biologists, physiologists, engineers and material scientists will accelerate establishment of reproducible human CNS models in vitro and will support more automated analysis of cellular, molecular and physiological responses.

Such effort will provide a range of novel platforms that will be valuable both for understanding human disease mechanisms and for performing initial medium/high throughput screenings of neuroactive compounds with increased power for predicting therapeutic potential, as well as neurotoxic effects, in humans. The advantages of this approach will not only be a significant reduction in the use of animals at the early stages of the drug discovery process, or in developmental neurotoxicology studies, but also a more in depth knowledge of the human disease mechanisms and a better understanding of interspecies differences. This in turn will help to develop/select better animal models for subsequent in vivo studies that are mandatory prior to clinical trials, that will underpin a more accurate interpretation of the results, hence reducing the risk of failure at advanced stages of therapeutic development. 


\section{References}

1. Muotri AR, Gage FH. Generation of neuronal variability and complexity. Nature 441(7097), 1087-1093 (2006).

2. Morrison B, Iii, Cullen DK, Laplaca M. In Vitro Models for Biomechanical Studies of Neural Tissues. In: Neural Tissue Biomechanics, Bilston LE (Ed.^(Eds).Springer Berlin Heidelberg 247-285 (2011).

3. Jakel RJ, Schneider BL, Svendsen CN. Using human neural stem cells to model neurological disease. Nat Rev Genet 5(2), 136-144 (2004).

4. Potashkin JA, Blume SR, Runkle NK. Limitations of animal models of Parkinson's disease. Parkinsons Dis 2011658083 (2010).

5. Clerc P, Lipnick S, Willett C. A look into the future of ALS research. Drug Discov Today 21(6), 939-949 (2016).

6. Chang R, Liu X, Li S, Li XJ. Transgenic animal models for study of the pathogenesis of Huntington's disease and therapy. Drug Des Devel Ther 9 2179-2188 (2015).

7. Blesa J, Przedborski S. Parkinson's disease: animal models and dopaminergic cell vulnerability. Front Neuroanat 8155 (2014).

8. Servadio M, Vanderschuren LJ, Trezza V. Modeling autism-relevant behavioral phenotypes in rats and mice: Do 'autistic' rodents exist? Behav Pharmacol 26(6), 522540 (2015).

9. Lawson CA, Martin DR. Animal models of GM2 gangliosidosis: utility and limitations. Appl Clin Genet 9 111-120 (2016).

10. Galli R, Pagano SF, Gritti A, Vescovi AL. Regulation of neuronal differentiation in human CNS stem cell progeny by leukemia inhibitory factor. Dev Neurosci 22(1-2), 86-95 (2000).

11. Oikari LE, Griffiths LR, Haupt LM. The current state of play in human neural stem cell models: what we have learnt from the rodent. OA Stem Cells 2(1), (2014). ** This useful review highlights similarities and differences between human and rodent neural stem cells

12. Ostenfeld T, Caldwell MA, Prowse KR, Linskens MH, Jauniaux E, Svendsen CN. Human neural precursor cells express low levels of telomerase in vitro and show diminishing cell proliferation with extensive axonal outgrowth following transplantation. Exp Neurol 164(1), 215-226 (2000). 
13. Baumann J, Gassmann K, Masjosthusmann S et al. Comparative human and rat neurospheres reveal species differences in chemical effects on neurodevelopmental key events. Arch Toxicol 90(6), 1415-1427 (2016).

14. Mourkioti F, Kustan J, Kraft P et al. Role of telomere dysfunction in cardiac failure in Duchenne muscular dystrophy. Nat Cell Biol 15(8), 895-904 (2013).

15. Basu N, Stamler CJ, Loua KM, Chan HM. An interspecies comparison of mercury inhibition on muscarinic acetylcholine receptor binding in the cerebral cortex and cerebellum. Toxicol Appl Pharmacol 205(1), 71-76 (2005).

16. Hopkins AM, Desimone E, Chwalek K, Kaplan DL. 3D in vitro modeling of the central nervous system. Prog Neurobiol 125 1-25 (2015).** This article provides an extensive review of strategies and tools for tissue-engineering 3D CNS systems and contains many useful references)

17. Walsh K, Megyesi J, Hammond R. Human central nervous system tissue culture: a historical review and examination of recent advances. Neurobiol Dis 18(1), 2-18 (2005).

18. Xie HR, Hu LS, Li GY. SH-SY5Y human neuroblastoma cell line: in vitro cell model of dopaminergic neurons in Parkinson's disease. Chin Med J (Engl) 123(8), 1086-1092 (2010).

19. Thomson JA, Itskovitz-Eldor J, Shapiro SS et al. Embryonic stem cell lines derived from human blastocysts. Science 282(5391), 1145-1147 (1998).

20. Schwartz MP, Hou Z, Propson NE et al. Human pluripotent stem cell-derived neural constructs for predicting neural toxicity. Proc Natl Acad Sci U S A 112(40), 1251612521 (2015).

21. Takahashi K, Tanabe K, Ohnuki M et al. Induction of pluripotent stem cells from adult human fibroblasts by defined factors. Cell 131(5), 861-872 (2007).

22. Prasongchean W, Ferretti P. Autologous stem cells for personalised medicine. New Biotechnology 29(6), 641-650 (2012).

23. Russo FB, Cugola FR, Fernandes IR, Pignatari GC, Beltrao-Braga PC. Induced pluripotent stem cells for modeling neurological disorders. World J Transplant 5(4), 209-221 (2015).

24. Wray S, Self M, Consortium NPSDI et al. Creation of an open-access, mutation-defined fibroblast resource for neurological disease research. PLoS One 7(8), e43099 (2012). 
25. Hedges EC, Mehler VJ, Nishimura AL. The Use of Stem Cells to Model Amyotrophic Lateral Sclerosis and Frontotemporal Dementia: From Basic Research to Regenerative Medicine. Stem Cells Int 20169279516 (2016).

26. Breslin S, O'driscoll $\mathrm{L}$. The relevance of using 3D cell cultures, in addition to 2D monolayer cultures, when evaluating breast cancer drug sensitivity and resistance. Oncotarget doi:10.18632/oncotarget.9935 (2016).

27. Eichler M, Jahnke HG, Krinke D et al. A novel 96-well multielectrode array based impedimetric monitoring platform for comparative drug efficacy analysis on $2 \mathrm{D}$ and 3D brain tumor cultures. Biosens Bioelectron 67 582-589 (2015).

28. Vagaska B. Modelling healthy and injured human central nervous system using human neural stem cells in 2D and 3D cultures. UCL Great Ormond Street Institute of Child Health $\mathrm{PhD}$ (2015).

29. Baker BM, Chen CS. Deconstructing the third dimension: how 3D culture microenvironments alter cellular cues. J Cell Sci 125(Pt 13), 3015-3024 (2012).

30. Knight E, Przyborski S. Advances in 3D cell culture technologies enabling tissue-like structures to be created in vitro. J Anat doi:10.1111/joa.12257 (2014).

31. Bar-Kochba E, Scimone MT, Estrada JB, Franck C. Strain and rate-dependent neuronal injury in a 3D in vitro compression model of traumatic brain injury. Sci Rep 630550 (2016).

32. Lyman WD, Tricoche M, Hatch WC, Kress Y, Chiu FC, Rashbaum WK. Human fetal central nervous system organotypic cultures. Brain Res Dev Brain Res 60(2), 155-160 (1991).

33. Verwer RW, Hermens WT, Dijkhuizen P et al. Cells in human postmortem brain tissue slices remain alive for several weeks in culture. Faseb $j$ 16(1), 54-60 (2002).

34. Andersson M, Avaliani N, Svensson A et al. Optogenetic control of human neurons in organotypic brain cultures. Sci Rep 624818 (2016).

35. Smith I, Silveirinha V, Stein JL et al. Human neural stem cell-derived cultures in threedimensional substrates form spontaneously functional neuronal networks. J Tissue Eng Regen Med doi:10.1002/term.2001 (2015).

36. Choi SH, Kim YH, Hebisch M et al. A three-dimensional human neural cell culture model of Alzheimer's disease. Nature 515(7526), 274-278 (2014). ** This study presents a model that can recapitulate significant aspects of Alzheimer's disease pathology, specifically amyloid- $\beta$ deposition, in a $3 \mathrm{D}$ human neural culture system. 
37. Cui QL, D'abate L, Fang $\mathrm{J}$ et al. Human fetal oligodendrocyte progenitor cells from different gestational stages exhibit substantially different potential to myelinate. Stem Cells Dev 21(11), 1831-1837 (2012).

38. Murphy SV, Atala A. 3D bioprinting of tissues and organs. Nat Biotech 32(8), 773-785 (2014).

39. Tasoglu S, Demirci U. Bioprinting for stem cell research. Trends in biotechnology 31(1), 10-19 (2013).

40. Gu Q, Tomaskovic-Crook E, Lozano R et al. Stem Cell Bioprinting: Functional 3D Neural Mini-Tissues from Printed Gel-Based Bioink and Human Neural Stem Cells (Adv. Healthcare Mater. 12/2016). Adv Healthc Mater 5(12), 1428 (2016). * This study highlights the promise of bioprinting for modelling the human nervous system.

41. Lancaster MA, Renner M, Martin C-A et al. Cerebral organoids model human brain development and microcephaly. Nature 501(7467), 373-379 (2013). ** This is one of the first papers reporting generation of human brain organoids and using it to study a disease that is not easily recapitulated in rodent models.

42. Eiraku M, Sasai Y. Self-formation of layered neural structures in three-dimensional culture of ES cells. Curr Opin Neurobiol 22(5), 768-777 (2012).

43. Cristini S, Alessandri G, Acerbi F et al. Three-dimensional self-organizing neural architectures: a neural stem cells reservoir and a system for neurodevelopmental studies. Tissue Eng Part C Methods 17(11), 1109-1120 (2011).

44. Eiraku M, Watanabe K, Matsuo-Takasaki M et al. Self-organized formation of polarized cortical tissues from ESCs and its active manipulation by extrinsic signals. Cell Stem Cell 3(5), 519-532 (2008). ** This is the first study showing that early stages of human corticogenesis can be mimicked in vitro in a 3D system using ESCs.

45. Lancaster MA, Knoblich JA. Generation of cerebral organoids from human pluripotent stem cells. Nat Protoc 9(10), 2329-2340 (2014). * The article provides a detailed protocol for the generation of human brain organoids and also discusses the limitations of the model.

46. Qian X, Nguyen HN, Song MM et al. Brain-Region-Specific Organoids Using Minibioreactors for Modeling ZIKV Exposure. Cell 165(5), 1238-1254 (2016). ** This study demonstrates the use of a miniaturised bioreactor for mainatining human brain organoids and how this new platform can be used to study the effect of Zika virus infection and putative antiviral compounds.

47. Mariani J, Coppola G, Zhang $\mathrm{P}$ et al. FOXG1-Dependent Dysregulation of GABA/Glutamate Neuron Differentiation in Autism Spectrum Disorders. Cell 162(2), 375-390 (2015). 
48. Wang L, Hou S, Han YG. Hedgehog signaling promotes basal progenitor expansion and the growth and folding of the neocortex. Nat Neurosci 19(7), 888-896 (2016).

49. Eiraku M, Takata N, Ishibashi $\mathrm{H}$ et al. Self-organizing optic-cup morphogenesis in three-dimensional culture. Nature 472(7341), 51-56 (2011).

50. Nakano $\mathrm{T}$, Ando $\mathrm{S}$, Takata $\mathrm{N}$ et al. Self-formation of optic cups and storable stratified neural retina from human ESCs. Cell Stem Cell 10(6), 771-785 (2012).

51. Bjornsson CS, Lin G, Al-Kofahi Y et al. Associative image analysis: a method for automated quantification of 3D multi-parameter images of brain tissue. $J$ Neurosci Methods 170(1), 165-178 (2008).

52. Cullen DK, Vukasinovic J, Glezer A, Laplaca MC. Microfluidic engineered high cell density three-dimensional neural cultures. J Neural Eng 4(2), 159-172 (2007).

53. Park J, Lee BK, Jeong GS, Hyun JK, Lee CJ, Lee S-H. Three-dimensional brain-on-achip with an interstitial level of flow and its application as an in vitro model of Alzheimer's disease. Lab on a Chip 15(1), 141-150 (2015).

54. Karimi M, Bahrami S, Mirshekari $\mathrm{H}$ et al. Microfluidic systems for stem cell-based neural tissue engineering. Lab Chip 16(14), 2551-2571 (2016).

55. Pamies D, Hartung T, Hogberg HT. Biological and medical applications of a brain-ona-chip. Exp Biol Med (Maywood) 239(9), 1096-1107 (2014).

56. Yang K, Han S, Shin Y et al. A microfluidic array for quantitative analysis of human neural stem cell self-renewal and differentiation in three-dimensional hypoxic microenvironment. Biomaterials 34(28), 6607-6614 (2013).

57. Yang Y, Gozen O, Watkins A et al. Presynaptic regulation of astroglial excitatory neurotransmitter transporter GLT1. Neuron 61(6), 880-894 (2009).

58. Frega M, Tedesco M, Massobrio P, Pesce M, Martinoia S. Network dynamics of 3D engineered neuronal cultures: a new experimental model for in-vitro electrophysiology. Sci Rep 45489 (2014).

59. Leijten J, Khademhosseini A. From Nano to Macro: Multiscale Materials for Improved Stem Cell Culturing and Analysis. Cell Stem Cell 18(1), 20-24 (2016).

60. Yin X, Mead BE, Safaee H, Langer R, Karp JM, Levy O. Engineering Stem Cell Organoids. Cell Stem Cell 18(1), 25-38 (2016).

61. Bible E, Qutachi O, Chau DY, Alexander MR, Shakesheff KM, Modo M. Neovascularization of the stroke cavity by implantation of human neural stem cells on VEGF-releasing PLGA microparticles. Biomaterials 33(30), 7435-7446 (2012). 
62. Carlson AL, Bennett NK, Francis NL et al. Generation and transplantation of reprogrammed human neurons in the brain using 3D microtopographic scaffolds. Nature Communications 710862 (2016).

63. Liang Y, Walczak P, Bulte JW. The survival of engrafted neural stem cells within hyaluronic acid hydrogels. Biomaterials 34(22), 5521-5529 (2013).

64. Ghuman H, Massensini AR, Donnelly J et al. ECM hydrogel for the treatment of stroke: Characterization of the host cell infiltrate. Biomaterials 91 166-181 (2016).

65. Giordano C, Albani D, Gloria A et al. Multidisciplinary perspectives for Alzheimer's and Parkinson's diseases: hydrogels for protein delivery and cell-based drug delivery as therapeutic strategies. Int J Artif Organs 32(12), 836-850 (2009).

Financial disclosure: The authors declare no conflict of interest.

Acknowledgements: We thank Great Ormond Street Hospital Children's Charity and Newlife Foundation for their support.

\section{Figure Legends}

Figure 1. Microenvironmental factors affecting cell behaviour. In vivo there are several factors that change temporally and spatially and substantially affect cellular behaviour within a tissue; some key ones are indicated. These factors need to be taken into account when designing 3-dimensional culture model with the aim of reproducing as accurately as possible a physiological cell behaviour within a tissues.

Figure 2. Summary of key steps and bottlenecks for the development of 3D in vitro models of human neural tissue. The central image shows a 3D culture of human embryonic neural stem cells neuronally differentiated in Matrigel and stained for ß3-tubulin (green), GFAP (red) and nuclei (blue). 
- Cell-cell communication

(polarity, signaling)

- Cell-matrix adhesions (polarity, mechanical inputs)
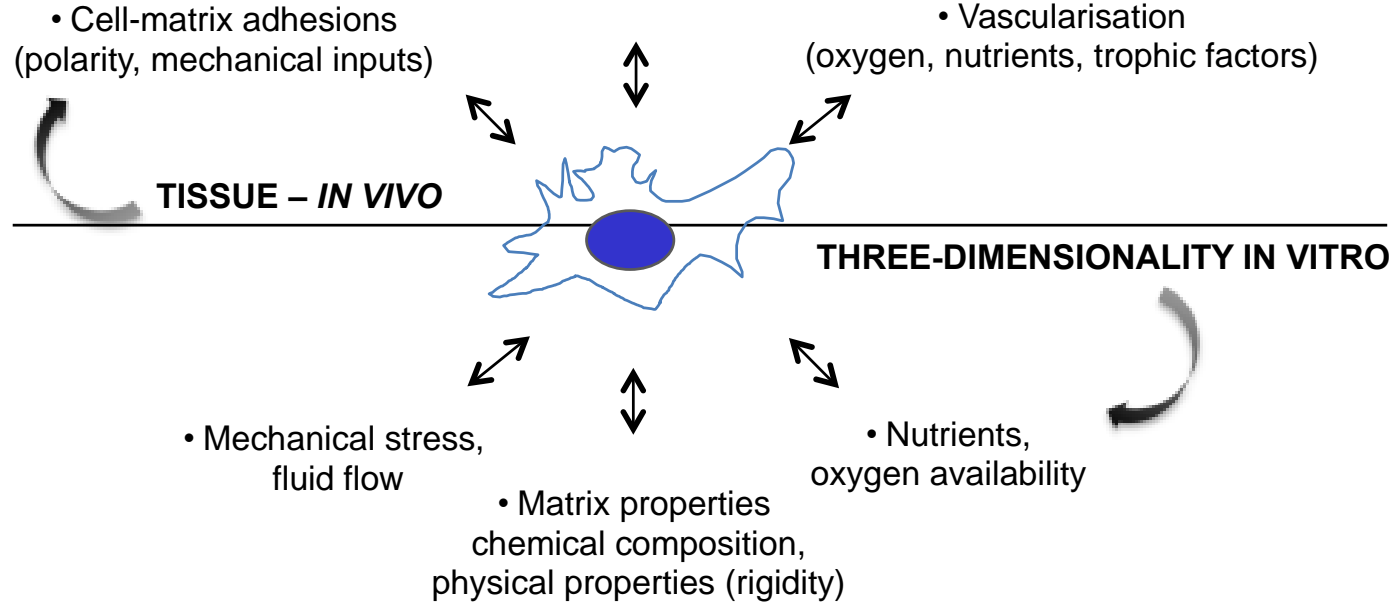

Figure 1. Microenvironmental factors affecting cell behaviour. In vivo there are several factors that change temporally and spatially and substantially affect cellular behaviour within a tissue; some key ones are indicated. These factors need to be taken into account when designing 3-dimensional culture model with the aim of reproducing as accurately as possible a physiological cell behaviour within a tissues. 


\section{3D environment:}

scaffold with defined structure, possibility of self-assembly

1. Cells:

source, type(s), density, differentiation protocols

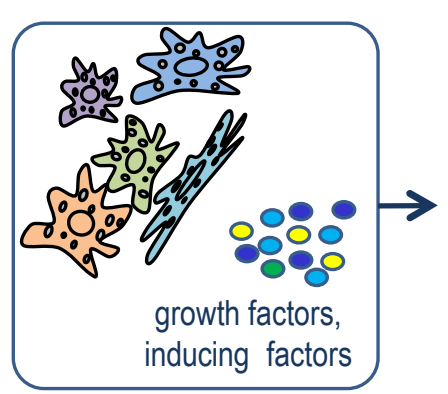

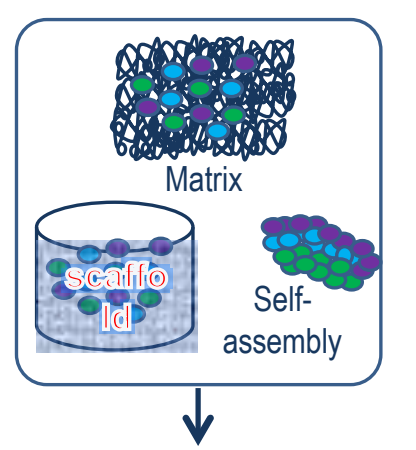
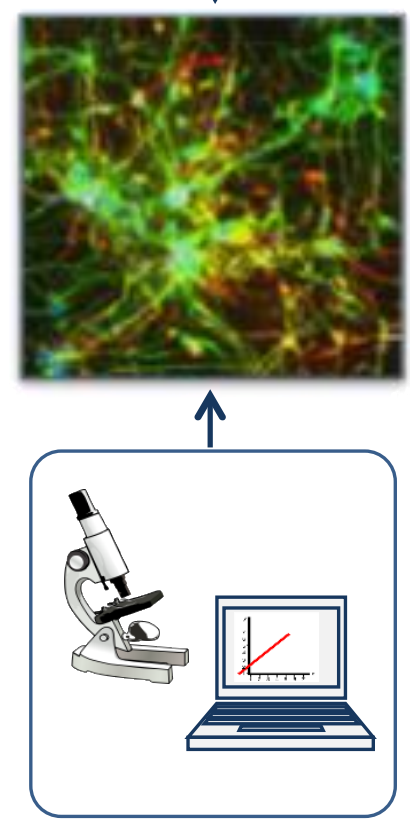

3. Environmental control: large scale, miniaturization

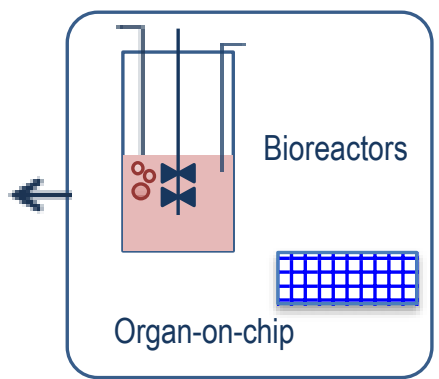

4. Tools for functional analysis:

imaging, biochemistry, electrophysiology

Figure 2. Summary of key steps and bottlenecks for the development of 3D in vitro models of human neural tissue. The central image shows a 3D culture of human embryonic neural stem cells neuronally differentiated in Matrigel and stained for B3-tubulin (green), GFAP (red) and nuclei (blue). 